\title{
Combining corneal crosslinking and phakic toric Implantable Collamer Lenses for the treatment of keratectasia: A case report
}

\author{
XIAO ZHANG, XIANG-CHEN TAO, ZHI-WEI LI, WEI-YAN ZHOU, PING MA, \\ CHUN-XIAO ZHANG, YU-MENG WANG and GUO-YING MU \\ Department of Ophthalmology, Shandong Provincial Hospital Affiliated to Shandong University, \\ Jinan, Shandong 250000, P.R. China
}

Received February 9, 2015; Accepted April 5, 2016

DOI: $10.3892 / \mathrm{etm} .2016 .3481$

\begin{abstract}
The present study reports the use of a phakic toric Implantable Collamer Lens (ICL) that improved the refraction correction of high myopia and astigmatism in a case of keratectasia following corneal cross-linking. A 31-year-old male was diagnosed with keratectasia 12 years after laser-assisted in situ keratomileusis (LASIK). Following LASIK, the manifest refraction was -3.50-2.25x90 [0.1 logarithmic expression (LogMAR) best corrected visual acuity (BVCA)] in the right eye and -8.00-3.50x175 (0.3 LogMAR BCVA) in the left eye, with a LogMAR uncorrected distance visual acuity (UDVA) of 0.8 and a 'counting fingers' value of $3 \mathrm{ft}$ (CF @3 ft) in the left and right eyes, respectively. Riboflavin/ultraviolet A light (UVA) corneal crosslinking (CXL) was conducted on both eyes. Seven months after cross-linking, LogMAR UDVA was 0.4, the manifest refraction was -2.75-2.50x85 and LogMAR BCVA was 0.1 in the right eye. In the left eye, LogMAR UDVA was CF@3 ft, the manifest refraction was -15.00 and LogMAR BCVA was 0.3. The corneal topography was stable 7 months after CXL. Phakic toric ICL was implanted to correct the refractive error, following which the LogMAR UDVA improved to 0.1 in the right eye and 0.3 in left eye, and visual acuity remained stable for 6 months after ICL implantation. In conclusion, combining riboflavin/UVA corneal cross-linking and phakic toric ICL implantation may be an alternative in the correction of high refractive error in patients with keratectasia.
\end{abstract}

Correspondence to: $\mathrm{Dr}$ Guo-Ying $\mathrm{Mu}$, Department of Ophthalmology, Shandong Provincial Hospital Affiliated to Shandong University, 324 Jingwu Road, Jinan, Shandong 250000, P.R. China

E-mail: mgyeyes@163.com

Key words: corneal crosslinking, riboflavin/ultraviolet A light, phakic toric implantable collamer lens, laser-assisted in situ keratomileusis, keratectasia

\section{Introduction}

For two decades, laser-assisted in situ keratomileusis (LASIK) has been used to correct myopia, as it is effective at improving visual acuity within a short time period. Typically, a 9-mm corneal flap is cut using a laser and, following laser treatment, the corneal flap is carefully repositioned (1). Keratectasia is one of the severe complications that may follow corneal refractive surgery, with an incidence between 0.04 and $0.6 \%$ following LASIK (2-4). The disease symptoms are the progressive steepening and thinning of the central cornea, with increasing myopia and astigmatism (5). Individuals experiencing keratectasia benefit from rigid gas permeable contact lenses (RGP) for refraction correction (6). However, contact lens intolerance may occur due to an irregularly shaped cornea or the complications of allergic conjunctivitis (7). Other surgical options to treat keratectasia include corneal cross-linking (CXL) combined with photorefractive keratectomy (PRK) in the early stages (8), and keratoplasty (9). Phakic toric Implantable Collamer Lenses (ICL) (STAAR Surgical, Nidau, Switzerland) have previously been used for their superiority in postoperative visual performance, as compared with corneal refractive surgeries such as LASIK, sub-Bowman's keratomileusis and Femto-LASIK (10), and for their safety and effectiveness in the correction of moderate-to-high ametropia (11-13). Numerous case reports have demonstrated that ICL corrects high refractive error in late stable keratoconus $(7,14)$. Riboflavin/ultraviolet A light (UVA) cross-linking is a technique for the treatment of keratoconus and keratectasia. UVA activates riboflavin into a triplet which, in turn, produces reactive oxygen species (ROS). ROS reacts with collagen fibril molecules in the cornea stroma and enhances the mechanical strength of the cornea to arrest the progression of keratoconus and keratectasia (15). The present study reports a case of keratectasia occurring 12 years following LASIK, and the use of riboflavin/UVA cross-linking to arrest disease progression, and ICL to correct high refractive error.

\section{Case report}

A 31-year-old man presented at the Department of Ophthalmology, Shandong Provincial Hospital Affiliated to Shandong University (Jinan, China) in November, 2013 with 

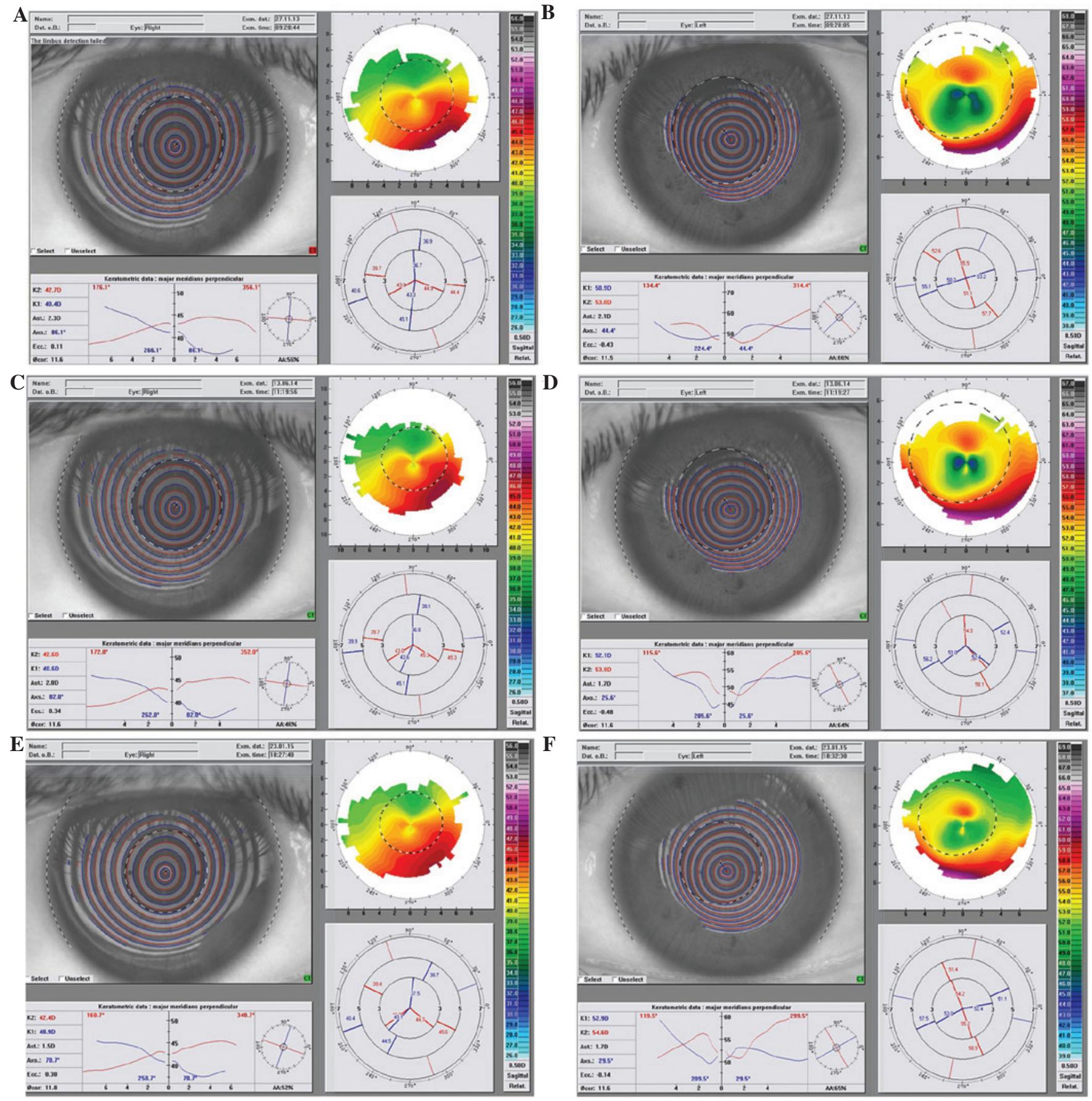

Figure 1. Corneal topography. Prior to corneal cross-linking (CXL) of the (A) right and (B) left eye; 7 month post-CXL of the (C) right and (D) left eye; 6 month post-ICL implantation of the (E) right and (F) left eye.

progressive vision loss in both eyes for 6 months, 12 years following LASIK. LASIK was performed at Yantai Economic \& Tech Development Zone Hospital (Yantai, China) in July, 2002. The pre-LASIK manifest refraction was $-2.25-0.75 \times 60$ in the right eye and $-1.50-0.25 \times 30$ in the left eye. The logarithmic expression (LogMAR) uncorrected distance visual acuity (UDVA) was 0.9 and 0.3 , and LogMAR best corrected visual acuity (BCVA) was -0.1 and 0.0 , for the right and left eyes respectively. The central corneal thickness (CCT; Tomey SP-3000; Tomey Corporation, Nagoya, Japan) was $548 \mu \mathrm{m}$ and $542 \mu \mathrm{m}$ in the right and left eyes, respectively, and corneal topography revealed no signs of keratoconus. The visual acuity of the right and left eyes was -0.1 and 0.0 respectively following LASIK, and remained stable for $>11$ years.

Slit-lamp examination revealed well-healed LASIK scars in both eyes and a peri-central corneal scar in the left eye. In the right eye following LASIK, LogMAR UDVA was 0.8 , the manifest refraction was -3.50-2.25x90 and LogMAR BCVA was 0.1 . In the left eye, LogMAR UDVA was a 'counting fingers' value of $3 \mathrm{ft}(\mathrm{CF} @ 3 \mathrm{ft})$, the manifest refraction was -8.00-3.50x175 and LogMAR BCVA was 0.3. Corneal tomography (Fig. 1; Allergo Topolyzer Vario; Wavelight $\mathrm{GmbH}$, Erlangen, Germany) revealed keratectasia with corneal astigmatism of $2.3 \mathrm{D}$ (Fig. 1A) in the right eye and $2.1 \mathrm{D}$ 
(Fig. 1B) in the left eye. The apex keratometric $(\mathrm{K})$ values were 42.88 D and 56.71 D, and CCT was $458 \mu \mathrm{m}$ and $437 \mu \mathrm{m}$ for the right and left eyes, respectively. The endothelial cell count (ECD) was acquired using a specular microscope (SP-3000P; Topcon Corporation, Tokyo, Japan) and was measured as 2,405 cells $/ \mathrm{mm}^{2}$ in the right eye; the ECD was not measured in left eye due to the corneal scar.

The present study was approved by the ethics committee of the Shandong Provincial Hospital Affiliated to Shandong University (Jinan, China) under the principles of the Helsinki Declaration (16), and written informed consent was obtained from the patient. CXL was performed in both eyes according to the Dresden protocol $(17,18)$ as follows: Following local anesthesia with proparacaine hydrochloride $0.5 \%$ eye drops (Alcaine; Alcon Laboratories, Inc., Fort Worth, TX, USA), the epithelium in the previous corneal flap (from LASIK) was removed by an excimer laser system with a wavelength of $193 \mathrm{~nm}$ (WaveLight EX500; WaveLight GmbH). In both eyes, the ablation diameter was $8.5 \mathrm{~mm}$ with a depth of $50 \mu \mathrm{m}$. For $30 \mathrm{~min}$ at intervals of $2 \mathrm{~min}, 0.1 \%$ riboflavin (Ricrolin; Sooft Italia S.p.A, Montegiorgio, Italy) was administered. When the cornea was fully penetrated with riboflavin (the yellow flare in the anterior chamber was monitored by slit-lamp examination), the cornea was illuminated with UVA (UV-X Illumination System, version 1000, UVXTM; IROCAG, Zurich, Switzerland) for $30 \mathrm{~min}$ at a working distance of $5 \mathrm{~cm}$, with irradiation of $3 \mathrm{~mW} / \mathrm{cm}^{2}$ and wavelength $370 \mathrm{~nm}$ (total dose $5.4 \mathrm{~J} / \mathrm{cm}^{2}$ ). During UVA illumination, riboflavin administration was continued at the same intervals. Following illumination, a soft contact lens was applied until the epithelium healed completely. Levofloxacin (Santen Pharmaceutical Co., Ltd., Osaka, Japan) and pranoprofen eye drops (SENJU Pharmaceutical Co., Ltd., Osaka, Japan) were used 4 times daily pre- and post-operation.

The patient was followed-up each month, and refraction and corneal topography remained stable until month 7 . In the right eye, LogMAR UDVA was 0.4 , the manifest refraction was -2.75-2.50x85 and LogMAR BCVA was 0.1. The apex K value decreased to $42.45 \mathrm{D}$ and corneal astigmatism was $2.0 \mathrm{D}$ (Fig. 1C). In the left eye, LogMAR UDVA was CF @3 ft, the manifest refraction was -15.00 and LogMAR BCVA was 0.3. The apex $\mathrm{K}$ value decreased to $55.52 \mathrm{D}$ and corneal astigmatism was $1.7 \mathrm{D}$ (Fig. 1D). ECD was 2,403 cells $/ \mathrm{mm}^{2}$ in the right eye; it was not measured in the left eye.

To correct the refractive error, ICLs were implanted in both eyes under local anesthesia using proparacaine hydrochloride $0.5 \%$ eye drops. Phakic toric ICL power was calculated according to the astigmatism decomposition method (11). One week prior to surgery, laser iridotomy was performed on both eyes using an ophthalmic photocoagulator (Quantel Medical, Cournon-d'Auvergne, France). The horizontal axis was marked at 90 and $270^{\circ}$ of the corneal limbus in the right eye in a sitting position. The pupil was dilated with tropicamide-phenylephrine eye drops (Santen Pharmaceutical Co., Ltd.). A temporal corneal incision and an inferior incision were performed, and Medical Hyaluronan Gel (Bausch \& Lomb) was injected into the anterior chamber. Phakic toric ICL (-7.5 D spherical power + 4.0 D cylinder power $\mathrm{x} 161^{\circ}$ axis in the right eye and $-22.5 \mathrm{D}$ spherical power in the left eye) were implanted in both eyes through the 3-mm temporal corneal incision using an injector cartridge supplied by the manufacturer (STAAR Surgical). The four haptics of ICL or toric ICL (T-ICL) were placed under the iris using a Battle ICL manipulator (Asico, Westmont, IL, USA). In the right eye, the phakic T-ICL cylinder axis was exactly aligned to the cylinder correction provided by a Mendez degree gauge (Katena Products, Inc., Denville, NJ, USA). Medical Hyaluronan Gel was completely washed out of the anterior chamber, and corneal incisions were closed using balanced salt solution (Alcon Laboratories, Inc.). At the end of the surgery, $2.5 \mathrm{mg}$ dexamethasone sodium (Jiangxi Xin'ganjiang Pharmaceutical Co., Ltd., Ji'an, China) was injected under the conjunctiva. Following the surgery, one drop of levofloxacin, pranoprofen and tobramycin-dexamethasone eye drops (Alcon Laboratories, Inc.) were used 4 times daily for 7 days.

Intraocular pressure remained normal at 13 and $12 \mathrm{mmHg}$ for the right and left eyes, respectively (normal range, $10-21 \mathrm{mmHg}$ ) following ICL implantation. One day post-operation, the LogMAR UDVA was 0.1 and 0.3 , and remained stable thereafter. After 1 week, the manifest refraction was $+0.50-0.75 \times 20$ in the right eye and $-0.75 \times 5$ in left eye. No intra- or post-operation complications were noted. At 6 months following ICL implantation, the LogMAR UDVA was 0.1 in the right eye and 0.3 in the left eye. The manifest refraction was $+0.50-1.25 \times 15$ and $-0.75 \times 20$, in the right and left eyes, respectively. The apex $\mathrm{K}$ value was $42.71 \mathrm{D}$ in right eye and 54.31 D in the left eye. Corneal astigmatism was $1.5 \mathrm{D}$ and 1.7 D (Fig. 1E and F), and CCT was $453 \mu \mathrm{m}$ and $432 \mu \mathrm{m}$, in the right and left eyes, respectively. ECD was 2,388 cells $/ \mathrm{mm}^{2}$ in the right eye; ECD was not measured in the left eye. The patient described good visual acuity, but experienced halo vision, particularly at night. In conclusion, ICL may be implanted for refraction correction and visual rehabilitation in patients with keratectasia when refraction and corneal topography are stable following cross-linking.

\section{Discussion}

Low residual stromal thickness and previous corneal risk factors, including pellucid marginal degeneration, thin corneal thickness and $<30$ years of age, may result in keratectasia (2). For LASIK candidates, careful preoperative examination is the key to prevent the occurrence of keratectasia. Riboflavin/UVA corneal collagen cross-linking increases the biomechanical stability of the cornea by forming new chemical bonds (19), and arrests the progression of the disease. In the present study, the patient experienced a slight flattening of the apex $\mathrm{K}$ value, and the range of keratectasia is decreased, following CXL.

In a previous study, Antonios et al (20) implanted ICL after CXL in a patient with keratoconus. Spherical and cylinder powers were demonstrated to be $-9.55 \pm 4.67$ and $2.38 \pm 1.29 \mathrm{D}$, respectively, 6-month after CXL, and decreased to $-1.63 \pm 0.95$ and $1.05 \pm 0.55 \mathrm{D} 24$-month following ICL implantation. Patients also exhibited a notable improvement in visual acuity. The present study described a patient with keratoectasia following LASIK, with spherical power up to $-15.00 \mathrm{D}$ in left eye 7-month post-CXL, and only $0.75 \mathrm{D}$ cylinder power in the left eye 6-month after ICL implantation. To the best of our knowledge, the present case report was the first to report CXL combined with ICL implantation for the correction of refractive keratectasia. 
At present, toric ICL is available with a cylindrical power up to $+6 \mathrm{D}$, and a spherical power between -3 and $-23 \mathrm{D}$, for myopia (21). Phakic toric ICL has been demonstrated to be effective for the correction of high myopic astigmatism in keratoconus eyes $(7,14)$. During phakic toric ICL implantation surgery, surgically induced astigmatism is less likely due to a $3-\mathrm{mm}$ corneal incision, thus the outcome is more predictable (21). In the current study, visual acuity was improved at 6 months following ICL implantation; however, the patient was confused by halo vision, particularly at night. This may be due to the pupils enlarging in the dark, as more light than previously penetrates through peripheral cornea. This suggests that although ICL can correct refractive error, further research is required in order to improve visual quality, particularly in the dark.

In conclusion, ICL implantation can be recommended when the cornea is stable since it can improve visual acuity. In the current study, refraction and topography were stable 7 months after CXL. ICL was then implanted, following which the cornea was stable and the visual outcome was improved at the 6 month follow-up. The results suggest that combining riboflavin/UVA corneal cross-linking and phakic toric ICL implantation may be an alternative for patients with keratectasia. Additional research is required in a larger number of patients, and long-term follow up should be conducted in order to confirm these findings.

\section{References}

1. Ivarsen A and Hjortdal J: Seven-year changes in corneal power and aberrations after PRK or LASIK. Invest Ophthalmol Vis Sci 53: 6011-6016, 2012.

2. Rad AS, Jabbarvand M and Saifi N: Progressive keratectasia after laser in situ keratomileusis. J Refract Surg 20 (Suppl 5): S718-S722, 2004

3. Ferreira TB, Marques EF and Filipe HP: Combined corneal collagen crosslinking and secondary intraocular lens implantation for keratectasia after radial keratotomy. J Cataract Refract Surg 40: 143-147, 2014.

4. Raiskup F and Spoerl E: Corneal crosslinking with riboflavin and ultraviolet A. Part II. Clinical indications and results. Ocul Surf 11: 93-108, 2013.

5. Kohlhaas M: Iatrogenic Keratectasia: A Review. Klin Monbl Augenheilkd 232: 765-772, 2015 (In German).

6. Bromley JG and Randleman JB: Treatment strategies for corneal ectasia. Curr Opin Ophthalmol 21: 255-258, 2010.
7. Kamiya K, Shimizu K, Ando W, Asato Y and Fujisawa T: Phakic toric Implantable Collamer Lens implantation for the correction of high myopic astigmatism in eyes with keratoconus. J Refract Surg 24: 840-842, 2008.

8. Kanellopoulos AJ and Binder PS: Management of corneal ectasia after LASIK with combined, same-day, topography-guided partial transepithelial PRK and collagen cross-linking: The Athens protocol. J Refract Surg 27: 323-331, 2011.

9. Dang TQ, Molchan RP, Taylor KR, Reilly CD, Panday VA and Caldwell MC: Novel approach for the treatment of corneal ectasia in a graft. Cornea 33: 310-312, 2014.

10. Huang D, Schallhorn SC, Sugar A, Farjo AA, Majmudar PA, Trattler WB and Tanzer DJ: Phakic intraocular lens implantation for the correction of myopia: a report by the American Academy of Ophthalmology. Ophthalmology 116: 2244-2258, 2009.

11. Sanders DR, Doney K and Poco M; ICL in Treatment of Myopia Study Group: United States food and drug administration clinical trial of the implantable collamer lens (ICL) for moderate to high myopia: Three-year follow-up. Ophthalmology 111: 1683-1692, 2004.

12. Sanders DR, Schneider D, Martin R, Brown D, Dulaney D, Vukich J, Slade S and Schallhorn S: Toric Implantable Collamer Lens for moderate to high myopic astigmatism. Ophthalmology 114: 54-61, 2007.

13. Gomez-Bastar A, Jaimes M, Graue-Hernández EO, Ramirez-Luquin T, Ramirez-Miranda A and Navas A: Long-term refractive outcomes of posterior chamber phakic (spheric and toric implantable collamer lens) intraocular lens implantation. Int Ophthalmol 34: 583-590, 2014.

14. Alfonso JF, Palacios A and Montes-Micó R: Myopic phakic STAAR collamer posterior chamber intraocular lenses for keratoconus. J Refract Surg 24: 867-874, 2008.

15. Zhang X, Tao XC, Zhang J, Li ZW, Xu YY, Wang YM, Zhang CX and Mu GY: A review of collagen cross-linking in cornea and sclera. J Ophthalmol 2015: 289467, 2015.

16. Botbol-Baum M: The Declaration of Helsinki in 2015 and the ethics of research in French-speaking Africa. J Int Bioethique 26: 87-101, 167, 2015 (In French)

17. Wollensak G, Spoerl E and Seiler T: Riboflavin/ultraviolet-a-induced collagen crosslinking for the treatment of keratoconus. Am J Ophthalmol 135: 620-627, 2003.

18. Kymionis GD, Mikropoulos DG, Portaliou DM, Voudouragkaki IC, Kozobolis VP and Konstas AG: An overview of corneal collagen cross-linking (CXL). Adv Ther 30: 858-869, 2013.

19. Raiskup F and Spoerl E: Corneal crosslinking with riboflavin and ultraviolet A. I. Principles. Ocul Surf 11: 65-74, 2013.

20. Antonios R, Dirani A, Fadlallah A, Chelala E, Hamade A, Cherfane C and Jarade E: Safety and visual outcome of visian toric icl implantation after corneal collagen cross-linking in keratoconus: Up to 2 years of follow-up. J Ophthalmol 2015: 514834, 2015.

21. Kamiya K, Shimizu K, Kobashi H, Komatsu M, Nakamura A, Nakamura $\mathrm{T}$ and Ichikawa K: Clinical outcomes of posterior chamber toric phakic intraocular lens implantation for the correction of high myopic astigmatism in eyes with keratoconus: 6-month follow-up. Graefes Arch Clin Exp Ophthalmol 249: 1073-1080, 2011. 